\title{
A Dual Axis Accelerometer Utilizing Low Doped Silicon Thermistor
}

\author{
Van Thanh Dau Non-member (Ritsumeikan) \\ Dzung Viet Dao Member (Ritsumeikan) \\ Masahiro Hayashida Non-member (Ritsumeikan) \\ Thien Xuan Dinh Non-member (Ritsumeikan) \\ Susumu Sugiyama Member (Ritsumeikan)
}

Keywords : Thermistor, convective accelerometer, dual axis, low-doped p-type silicon, thermal stress

\section{Working Principle}

Micro accelerometers such as the capacitive, piezoresistive, piezoelectric, optical were researched and fabricated for a long decade. Most of them need seismic mass and therefore they have disadvantage of fragileness. Thermal convective accelerometer without the mass can overcome the problem of those "mechanical" sensors. This paper presents a new design of thermal convective accelerometer which can detect two components of the acceleration. Different with the reported clambed-clambed thermal sensing structure, our sensor utilize a free-free beam so that the thermal stress is reduced. By using numerical method, the sensor parameters are optimized. Other characteristics such as frequency response, shock resistance, noise problem are also deeply investigated. The sensor has been fabricated by MEMS process.

The working principle is based on thermo resistive effect in silicon. The sensor with total dimension of $2 \times 2 \mathrm{~mm}^{2}$ consists of one heat source at the center, surrounded by a micro thermal sensing element. The sensor then will be packaged in order to form a chamber of $5 \times 5 \times 2 \mathrm{~mm}^{3}$. The micro sensing element is formed by four silicon thermistor wires, each has dimension of $140 \times 6 \times 5 \mu \mathrm{m}^{3},(\mathrm{~L} \times \mathrm{W} \times \mathrm{T})$, arranged in a ring-like shape. When the sensor is subjected to acceleration, the heat stream will move in the reverse direction of inertial force, causing the temperature deflection at one pair of opposite thermistors. And as a result, the resistance of these thermistors is changed oppositely; this creates the output voltage through the Wheatstone bridge. The dimensions of the cavity and the position of the sensing element are decided

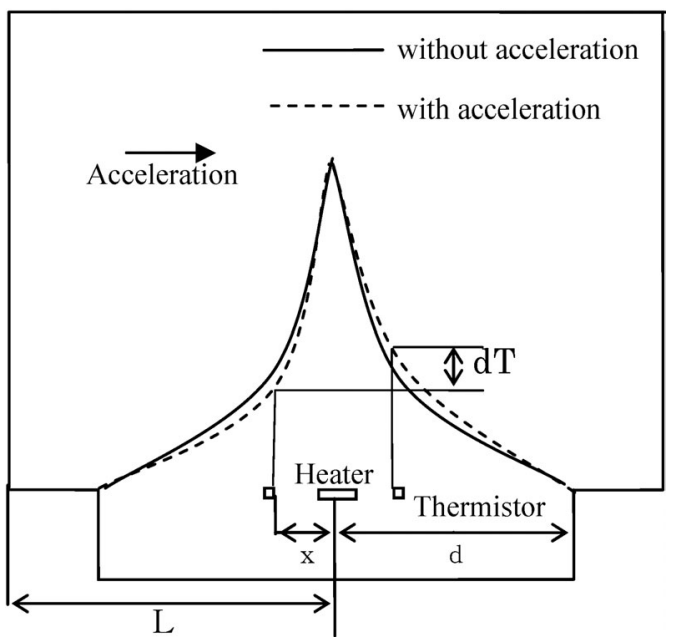

Fig. 1. Schematic view shows the sensor working principle based on the sensitivity simulation. Three most important dimensions $\mathrm{L}, \mathrm{d}$ and $\mathrm{x}$ (as shown in Fig. 1) are found to have strong effect to the sensitivity of the sensor. This is achieved from the thermal-flow simulation.

\section{Theoretical Characteristics and Fabrication}

The sensitivity of the sensor was simulated and earned the nonlinearity of $0.23 \%$. At room temperature, the working temperature of the heat source and of the thermal sensing element is $200^{\circ} \mathrm{C}$ and $83^{\circ} \mathrm{C}$, respectively. By using coupled field analysis of electric-thermal-structure, the power consumption of the sensor was $12.5 \mathrm{~mW}$, the shock resistance was achieved up to $3.8 \times 10^{5} \mathrm{~g}$ and the thermal stress was also guaranteed. The sensitivity of the sensor depends on the temperature of the heat source. The simulation of the frequency response, which is disadvantage of thermal convective accelerometer, and the bandwidth of $250 \mathrm{~Hz}$ was achieved at $-3 \mathrm{~dB}$. The resolution of the sensor based on noise analysis is calculated to be $0.1 \mathrm{mg}$

The sensor has been fabricated from SOI wafer by conventional MEMS technology. During the fabrication processes, polyimide was used in order to protect the thin-long structures from thermal and mechanical attacks. Fig. 2 shows a fabricated sensor with resistance of each p-type Si thermistor was measured to be $12 \mathrm{k} \Omega$. The resistance was linearly proportional to temperature applied to the thermistor, and the TCR of $6000 \mathrm{ppm} /{ }^{\circ} \mathrm{C}$ was obtained at impurity concentration of $6 \times 10^{16} \mathrm{~cm}^{-3}$. The sensor was hermetically packaged and calibrated.

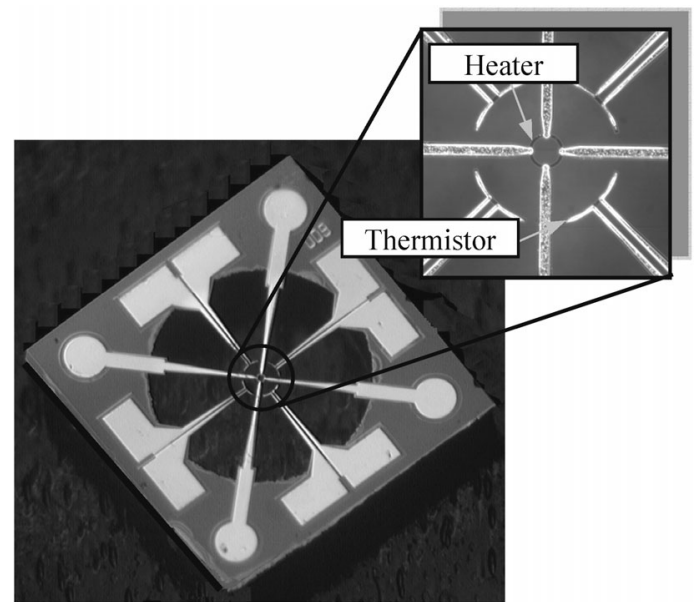

Fig. 2. Micrograph of the $2 \times 2 \times 0.4 \mathrm{~mm}^{3}$ sensing chip consists of four thermistors (arranged in a ring-like shape) and a heater 


\title{
A Dual Axis Accelerometer Utilizing Low Doped Silicon Thermistor
}

\author{
Van Thanh Dau* Non-member \\ Dzung Viet Dao* Member \\ Masahiro Hayashida* Non-member \\ Thien Xuan Dinh* Non-member \\ Susumu Sugiyama* Member
}

\begin{abstract}
This paper presents the development of a dual axis convective microaccelerometer, whose working principle is based on the convective heat transfer and thermo-resistive effect of lightly-doped silicon. Different with developed convective accelerometer, the sensor utilizes a novel structure of the sensing element which can reduce 93\% of thermal-induced stress. Moreover, the thermistors are made from low-doped p-type silicon, which has the TCR higher than that of metals and poly-silicon convective accelerometer. By using numerical method, the chip dimensions and the package size are optimized. The sensitivity of the sensor was simulated; other characteristics such as frequency response, shock resistance, noise problem are also deeply investigated. The sensor has been fabricated by MEMS process and characterized by experiments.
\end{abstract}

\section{Keywords : Thermistor, convective accelerometer, dual axis, low-doped p-type silicon, thermal stress}

\section{Introduction}

Many types of micro accelerometer were involved in these decades and are not only used for cars or airplanes, but also for cell phone and games. Demands for compact, low cost and high performance micro accelerometers keep increasing in many fields including automobile industry, robotic systems, and entertainment devices. In general, accelerometers utilizing the proof mass to sense the applied acceleration. The proof mass, however, has some disadvantages of low shock resistance, high possibility of fragility, complex fabrication process, and so on.

Recently, micro thermal convective accelerometer utilized the thermal bubble instead of the mass is widely studied ${ }^{(1)-(6)}$ since they can overcome the disadvantages of the ordinary "mechanical" accelerometers $^{(7)-(8)}$. This paper presents the optimal design and fabrication of a dual axis convective micro accelerometer utilizing a novel structure for micro thermistors. The sensor can detect two components of acceleration simultaneously. The thermistors are made from low-doped p-type silicon, which has TCR much higher than that of metals and poly-silicon.

Most of convective accelerometers reported recently in MEMS have only one degree of freedom and based on the thermo-resistive effect in metal or poly-silicon. Moreover, the sensing thermistors were not optimized in term of thermal stress. The clamped-clamped beam structure was widely used for the sensing element, and therefore, thermal stress occurred when the temperature is changed. Thermal stress in a p-type Si thermistor causes out-of-plane deformation and sensitivity reduction due to opposite influences of thermo-resistive and piezoresistive effects.

By using numerical simulation, the sensor parameters have been comprehensively studied and optimized. Other characteristics, such as frequency response, shock resistance, noise problem are also deeply investigated. The sensor has been fabricated from SOI

\section{* Ritsumeikan University,} 1-1-1 Noji Higashi, Kusatsu, Shiga, 525-8577, Japan wafer by MEMS process. Performance of the proposed accelerometer was simulated and characterized by experiments.

\section{Working Principle}

The sensor configuration consists of a $2 \times 2 \mathrm{~mm}^{2}$ sensing chip packaged in a hermetical chamber (Fig. 1). The sensing chip is formed by one heater at the center surrounding by four micro thermistors. Each thermistor, has the shape of a circular curve, is free at both ends and fixed to the arm in the middle. The dimensions of each thermistor are $140 \times 6 \times 5 \mu \mathrm{m}^{3},(\mathrm{~L} \times \mathrm{W} \times \mathrm{T})$.

When no acceleration is applied, the heater creates a symmetrical temperature profile inside the chamber so that no temperature difference on the thermistor is appeared. When the

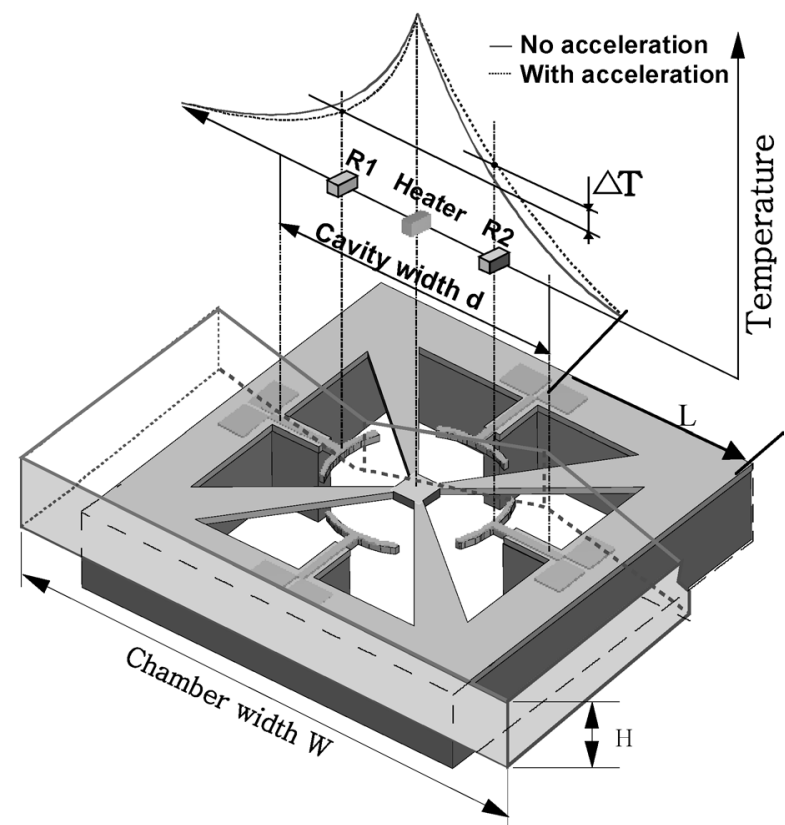

Fig. 1. Schematic view of the sensor 
sensor is subjected to acceleration, the thermal bubble will move in the direction of the applied acceleration, causing the temperature of one thermistor increases while decreases temperature of the opposite one. Due to the thermo-resistive effect of silicon, the resistance of these thermistors is changed oppositely as below.

$$
R_{1,2}=R_{0}\left(1 \pm \alpha \frac{1}{2} \Delta t\right)
$$

where $R_{1}$ and $R_{2}$ are the resistances of the opposite thermistors. $R_{0}, \alpha, \Delta t$ are resistance of the thermistor at room temperature, temperature coefficient of resistance (TCR) and deflection of the temperature, respectively.

This resistance change is then converted to output voltage change by the Wheatstone bridges and is expressed as follow.

$$
\begin{aligned}
& V_{\text {output }}=\frac{R_{1}-R_{2}}{2\left(R_{1}+R_{2}\right)} V_{\text {input }} \\
& V_{\text {ouput }}=\frac{\alpha \Delta t}{4} V_{\text {input }}
\end{aligned}
$$

\section{Simulation and Design}

Different from seismic-based accelerometer, in convective accelerometers the performance strongly depends on the cavity size (the space under the thermistors), the chamber size (the space upper the thermistors), the position of the thermistors, and the temperature of the heater. To decide the space of the sensor, the cavity is first optimized since the cavity size is comparable with the heater. The small cavity size will significantly restrain the flow pattern and drop the performance. The commercial finite element model software ANSYS has been employed to simulate the incompressible laminar flow inside the sensor using a

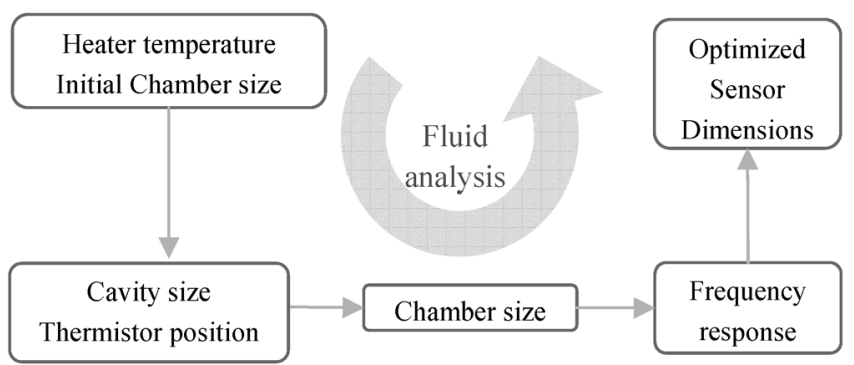

Fig. 2. Flow chart of the analysis

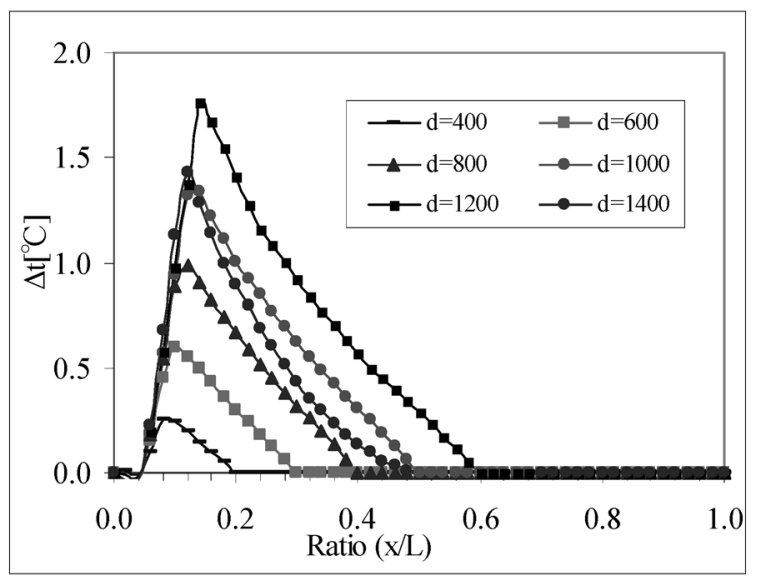

Fig. 3. The temperature deflections $\Delta T$ between two opposite points at both sides of the heater. Applied acceleration is $10 \mathrm{~g}$ ( $\mathrm{g}$ is gravity acceleration) three-dimensional model, the deflections of temperature at different positions around the heater corresponding to various cavity sizes are shown in Fig. 3. Simulation shows that the cavity diameter should be $1200 \mu \mathrm{m}$ and thermistor has the optimum position at $x / L=0.18$, where $L$ and $x$ are respectively the half of the chip size and the distance from the heater to each thermistor. The sensing chip is then fabricated with the decided dimensions.

Figure 4 shows the relation between sensor sensitivity versus the size of the packaged chamber at different temperatures of the heater. The sensitivity of the sensor increases while increasing the volume of the chamber, i.e. $W$ and $H$. However, the larger space will reduce the frequency response of the sensor and limit the sensor's application. Based on consideration of these factors, the size of the accelerometer is decided to be $5000 \times 5000 \times 2000 \mu \mathrm{m}^{3}$, $(W \times W \times H)$. The sensor with larger packaged size can only lightly improve the sensitivity.

Frequency response was simulated for the packaged sensor by applied square wave of input acceleration. The result was shown in Fig. 5 and the bandwidth of the sensor was $250 \mathrm{~Hz}$ at $-3 \mathrm{~dB}$. The response of the tiny thermistor, which is reported to be in micro

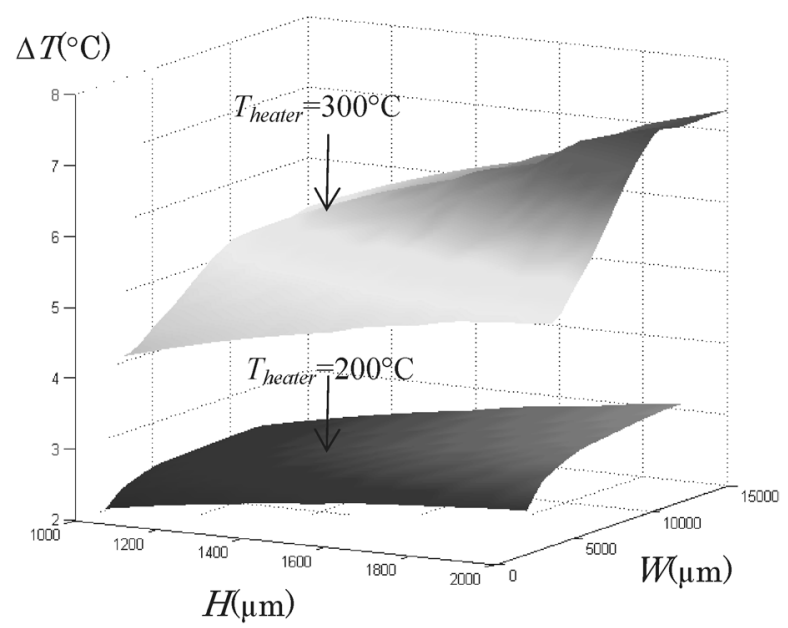

Fig. 4. Relation between temperature deflections (sensitivity) versus the size of the packaged chamber at different temperatures of the heater. Applied acceleration is $10 \mathrm{~g}$ ( $\mathrm{g}$ is gravity acceleration)

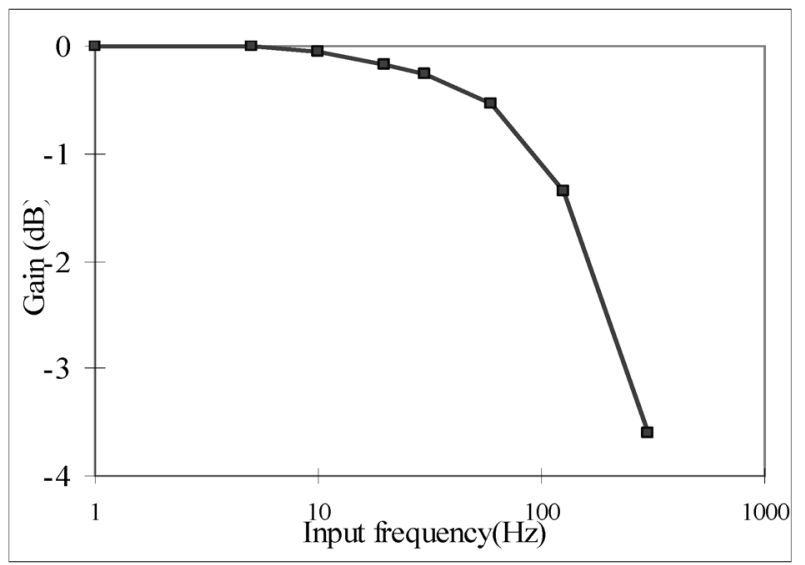

Fig. 5. Frequency response of the sensor corresponding to the chamber size of $5000 \times 5000 \times 2000 \mu \mathrm{m}^{3}$ (W x W x $\mathrm{H})$. The time constant of the thermistor is not included 
second scale ${ }^{(9)}$, is not included.

\section{Structure Analysis}

Most of the thermal convective accelerometers developed so far utilize the clamped-clamped structure for the sensing element ${ }^{(1)-(6)}$. Therefore, as the temperature is changed, the thermal stress will appear because of restriction in thermal-deformation of the structure. Our sensor uses a new structure that allows the thermistor to expand freely when temperature increases. Therefore, theoretically, the thermal stress is not built in the structure. However, due to the stacked-layers structure $\left(\mathrm{Al}-\mathrm{SiO}_{2}-\mathrm{Si}\right)$ of the thermistor, and with the mismatch of thermal coefficient of expansions between each layer, some thermal stress will be occurred. Thermal stress in a p-type silicon thermistors will cause the sensitivity reduction due to opposite influences of thermo-resistive and piezoresistive effects ${ }^{(10)}$.

Numerical simulations by means of coupled field analysis of electric-thermal-structure were performed to clarify this problem. Working temperature of the thermistors at acceleration-free state is $83^{\circ} \mathrm{C}$, the heat source was heated up to $200^{\circ} \mathrm{C}$ corresponding to a power consumption of $12.5 \mathrm{~mW}$. The results show that the average thermal stress of the clamped-clamped thermistor increases up to $24 \mathrm{MPa}$ when the thermistor is heated from room temperature of $25^{\circ} \mathrm{C}$, while that of the new structure was $2.2 \mathrm{MPa}\left(0.038 \mathrm{MPa} /{ }^{\circ} \mathrm{C}\right)$, i.e. $93 \%$ of thermal-induced stress was reduced. Moreover, the sensor can also achieved the shock resistance up to $3.8 \times 10^{5} \mathrm{~g}$ ( $\mathrm{g}$ is gravity acceleration).

\section{Noise and Resolution of the Accelerometer}

There are mainly two typical noise source existing in the thermistor wire, including Johnson-noise and Hooge's noise (or 1/f noise).

Johnson noise is due to random motion of carriers in any electrical conductor and is described as:

$$
V_{J n(r m s)}=\sqrt{4 K_{B} T R B}
$$

Where $K_{B}$ is Boltzman's constant $\left(1.38 \times 10^{-23} \mathrm{~J} / \mathrm{K}\right), T$ is temperature $(\mathrm{K}), R$ is resistance of the thermistor, and $B$ is measurement bandwidth (Hz). Accordingly, the Johnson noise for measurement bridge is calculated to be $V_{J n}=0.19 \mu \mathrm{V}$ when sensor is working at room temperature.

$1 / f$ noise is empirically determined noise which is dependent on the total number of carriers, according to the equation ${ }^{(11)}$ :

$$
V_{1 / f(r m s)}^{2}=\frac{\alpha V_{\mathrm{in}}^{2}}{N} \ln \left(\frac{f_{\max }}{f_{\min }}\right)
$$

Where $V_{\text {in }}$ is bias voltage across a thermistor with total number of carriers $N$, and $f_{\max }$ and $f_{\min }$ are upper and lower limit of measurement frequency, respectively. $\alpha$ is a dimensionless parameter called Hooge parameter. For semiconductor, $\alpha$ was found to be $10^{-7(12)}$. For a constant doping concentration, the number of carriers $N$ is proportional to the volume of the thermistor. Finally, $1 / f$ noise voltage in each measurement bridge can be calculated to be $V_{I / f}=0.42 \mu \mathrm{V}$.

Total noise voltage: The total noise voltage in the accelerometer for one measurement bridge is:

$$
V_{\text {Noise }}=\sqrt{\left(V_{J n}\right)^{2}+\left(V_{1 / f}\right)^{2}}=0.45 \mu \mathrm{V} \text {. }
$$

The resolution of an accelerometer determines the minimum acceleration that can be measured. Resolution is defined as the

noise divided by the sensitivity. Accordingly, resolution of the accelerometer is $0.1 \mathrm{mg}$ at the heater temperature of $200^{\circ} \mathrm{C}$.

\section{Fabrication}

The fabrication of the sensing chip begins with a silicon-on-insulator (SOI) wafer with a p-type device layer of $2.5 \mu \mathrm{m}$, and a resistivity of $0.35 \mathrm{Ohm}-\mathrm{cm}$. Next, a $0.3 \mu \mathrm{m}$-thick $\mathrm{SiO}_{2}$ is formed by thermal oxidization to create the insulating layer. The contact holes are opened (Fig. 6(a)) and p+ diffusion is performed to improve the metal-semiconductor contact (Fig. 6 (b)). Aluminum wires of $0.15 \mu \mathrm{m}$ thickness for interconnection are made by vacuum evaporation, photolithography and Al etching. Then, sintering is carried out to make the Ohmic contact between the $\mathrm{Al}$ wires and the device layer through the contact holes (Fig. 6(c)). The thermistors are patterned by photolithography and ICP-RIE (Fig. 6(d)). In order to protect the thin-long structures from mechanical damages in the following processes, the thermistors are covered by photoresist (on frontside) and a layer of polyimide is deposited on top. The backside is etched by using ICP-RIE to create the structure of the thermistor (Fig. 6(e)). Next,

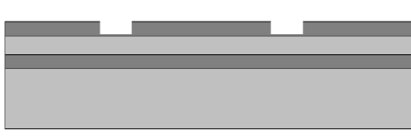

(a)

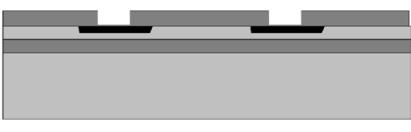

(b)

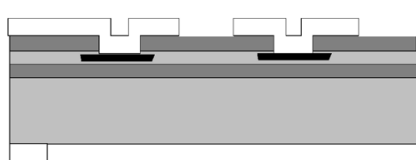

(c)

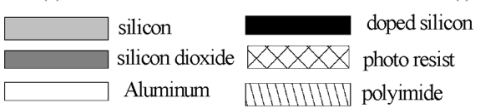

Fig. 6. Fabrication process of the sensing chip

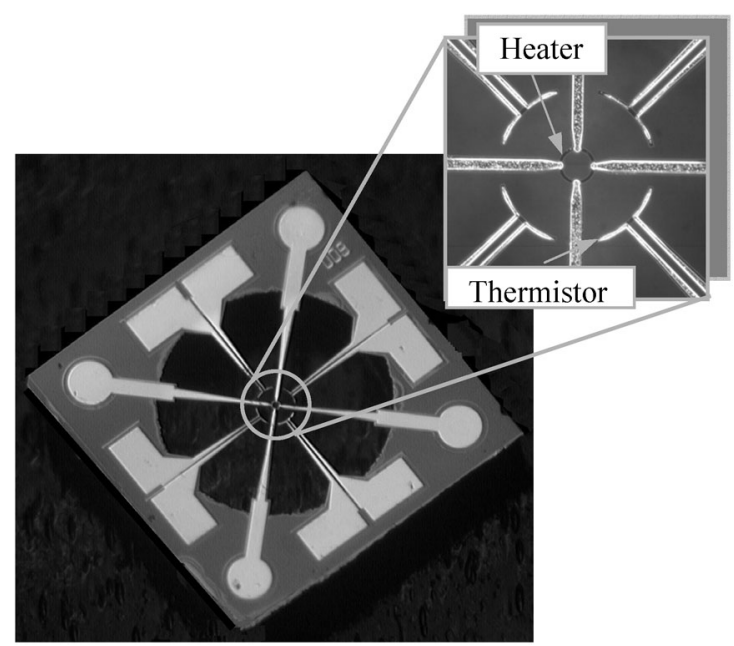

Fig. 7. Micrograph of the $2 \times 2 \times 0.4 \mathrm{~mm}^{3}$ sensing chip consists of four thermistors (arranged in a ring-like shape) and a heater. Dimension of the heater is $\Phi 50 \mathrm{x} 5 \mathrm{x} 5 \mu \mathrm{m}^{3}$ and the thermistor is $140 \times 6 \times 5 \mu \mathrm{m}^{3}$ 


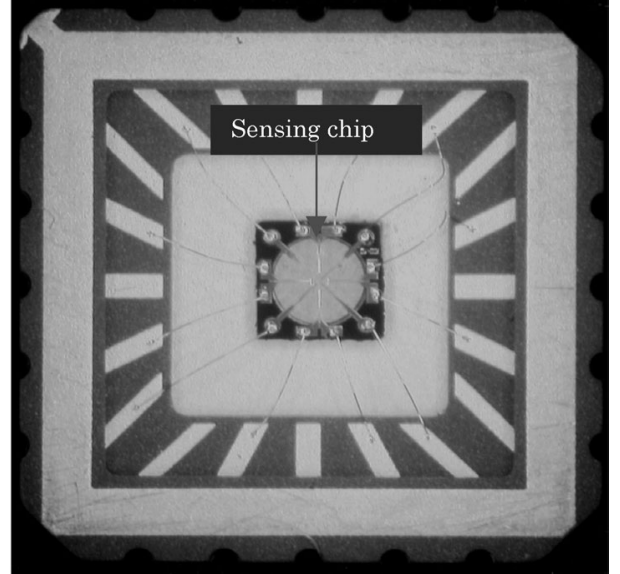

Fig. 8. View of hermetically packaged sensor with glass cover. Chamber size is $5000 \times 5000 \times 2000 \mu^{3}$ $(\mathrm{W} \times \mathrm{W} \times \mathrm{H})$

the buried oxide is removed by HF vapor. The polyimide/photoresist protection layers are finally peeled off by removing the underneath photoresist (Fig. 6(f)). Fig. 7 is the micrograph of the fabricated sensing chip for the thermal convective accelerometer.

After fabrication process, the sensing element is then aligned and bonded on a bottom part of the case. The connection between the on-chip bridges to off-chip circuit is done by gold wire, which has the diameter of $25 \mu \mathrm{m}$. During the package process, the symmetry needs to be maintained. Currently, air with pressure of latm is used as the flow ambient for thermal convective accelerometer. The sensor is finally sealed with a glass cover and an image of a packaged sensor is shown in Fig. 8.

\section{Experiment results}

The TCR of the thermistor is tested by placing the sensing chip in the oven and increasing the oven temperature from $-30^{\circ} \mathrm{C}$ to $70^{\circ} \mathrm{C}$. The data is then drawn in Fig. 9. The resistance is linearly proportional with the temperate and a TCR value of $6000 \mathrm{ppm} /{ }^{\circ} \mathrm{C}$ is achieved.

In this paper, the measurement circuit was formed as shown in Fig. 10. The gain of the amplifier is 500 .

To test the performance, the packaged accelerometer is placed on a turntable and centripetal acceleration is used as applied input. The sensor was characterized at $\pm 5 \mathrm{~g}$ (g is gravity acceleration). The output voltage versus applied acceleration was measured, and the result is shown in Fig. 11. The sensor sensitivity was $10 \mathrm{mV} / \mathrm{g}$, and a nonlinearity of $0.23 \% \mathrm{FSO}$ was realized.

\section{Conclusion}

A dual axis thermal convective accelerometer was optimized. The new sensing structure successfully reduced the thermal stress of the thermistor up to $93 \%$ of that of "ordinary" clamped-clamped thermal sensing elements.

The performance of convective sensor is strongly affected by the on chip cavity dimension and package chamber size. The optimized values of the on-chip dimensions are calculated. In order to package the fabricated sensing chip, the relation between chamber size and the sensor's performance at different temperature of the heater is simulated. The accelerometer is then packaged by compromising the sensitivity and the bandwidth of

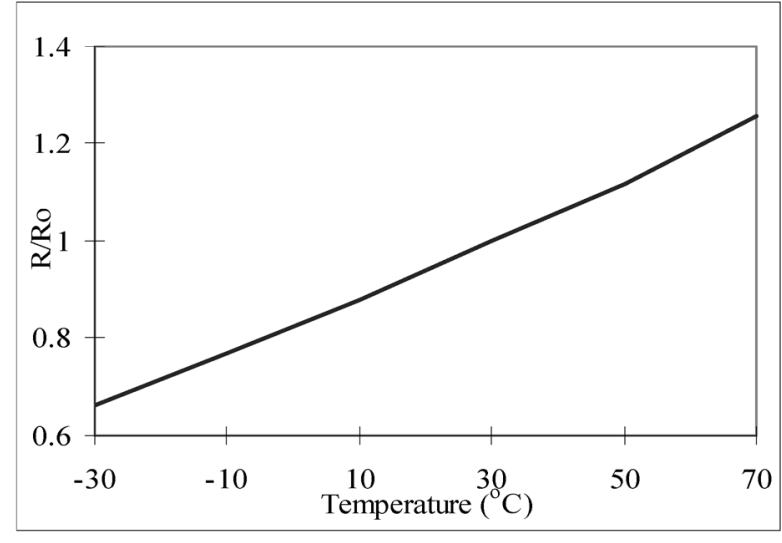

Fig. 9. TCR of a lightly-doped p-type silicon thermistor. Resistivity of the thermistor is $0.35 \mathrm{Ohm}-\mathrm{cm}$. $\mathrm{R}$ and $\mathrm{R}_{0}$ are respectively the resistances at test and at room temperatures

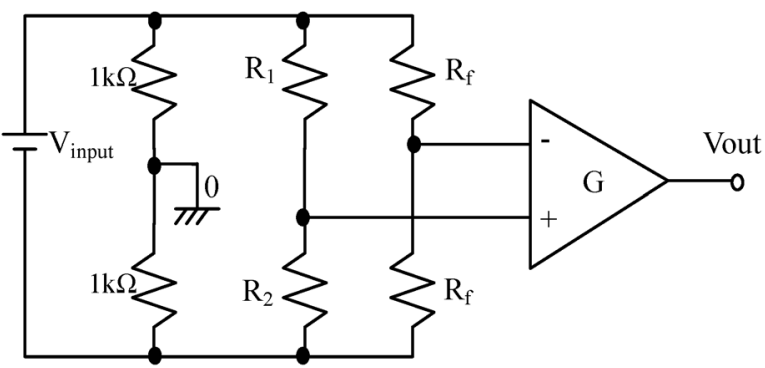

Fig. 10. Measurement circuit for thermal accelerometer, two $1 \mathrm{k} \Omega$ resistors are used in order to apply an value of $\left(\mathrm{V}_{\text {input }} / 2\right)$ on the thermistors

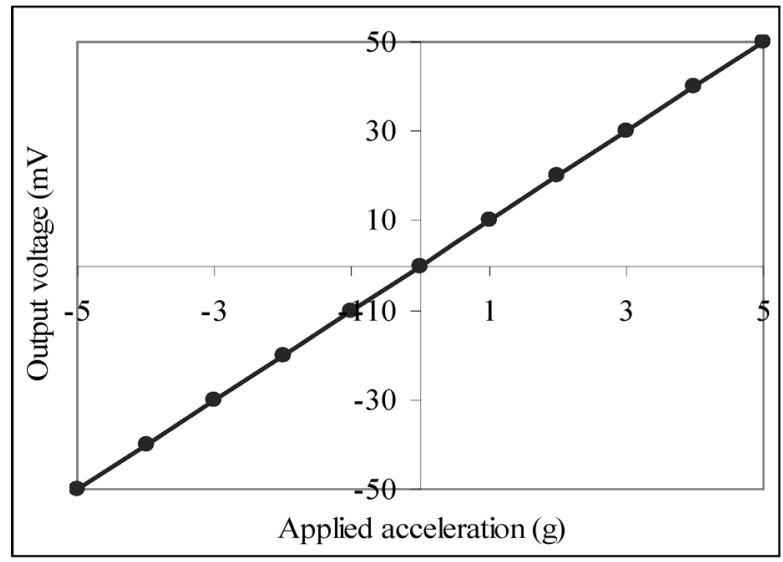

Fig. 11. The measured sensitivity of the sensor. Measurement condition: $V_{\text {input }}=1 \mathrm{~V}$ and heater power $=$ $12.5 \mathrm{~mW}$

frequency.

The performance of the sensor has been tested by experiments and high linearity was achieved. However, due to the imperfectness in packaging, the experimental result is still smaller than simulation. Different with the seismic-based accelerometer, the sensitivity of the convective accelerometer can be enhanced by increasing the Grashof number ${ }^{(4)}$, i.e by choosing the working medium that has small kinematic viscosity or by increasing the temperature of the heater.

The dynamic viscosity of a gas increases with an increase of 
temperature ${ }^{(13)}$. As the sensor is hermitically packaged the working gas kinematic viscosity, which is defined by the ratio of dynamic viscosity and the density, will also increase with the ambient temperature. The sensitivity of the sensor therefore decreases with the increase of temperature.

(Manuscript received Oct. 23, 2005, revised Nov. 30, 2005)

\section{References}

(1) J W van Honschoten et al. : "Analytic model of a two wire thermal sensor for flow and sound measurement", J. Micromech. Microeng., Vol.14, pp.1468-1477 (2004)

(2) Ke-Min Liao et al. : "A Novel Thermal-Bubble-Based Micromachined Accelerometer", Transducers, Vol.05, pp.519-522 (2005)

(3) F. Mailly et al. : "Effect of gas pressure on the sensitivity of a micromachined thermal accelerometer", Sensors and Actuators., Vol.109, pp.88-94 (2003)

(4) X B Luo et al. : "Study on linearity of a micromachined convective accelerometer", Micromachine Eng., Vol.65, pp.87-101 (2003)

(5) N.Sabate et al. : "Multi-range silicon micromachined flow sensor", Sensors and Actuators A, Vol.110, pp.282-288 (2004)

(6) M. Baroncini et al. : "Thermal characterization of a microheater for micromachined gas sensors", Sensors and Actuators., Vol.115, pp.8-14 (2004)

(7) L. C. Spangler and C. J. Kemp : "ISAAC: integrate silicon automotive accelerometer", Sens. Actuators A, Vol.54, pp.523-529 (1996)

(8) T. Mineta, S. Kobayashi, Y. Watanabe, S. Kanauchi, I. Nakagawa, E. Wuganuma, and M. Esashi : "Three-axis capacitive accelerometer with uniform axial sensitivities", J. Micromech. Microeng., Vol.6, pp.431-435 (1996)

(9) F. Jiang, Yu-Chong Tai, C. M. Ho, and Wen J. Li : "A micromachined Polysilicon Hot-Wire Anemeter", Solid-State Senor and Actuator Workshop, pp.264-267 (1994)

(10) D. V. Dao : "Study on Silicon Piezoresistive Six DOFs Micro Forced Moment Sensors and Application to Fluid Mechanics", Doctoral Thesis, Ritsumeikan Univ., Japan (2003)

(11) F. N. Hooge : "1/f noise is no surface effect", Physical lett. A, Vol.29, pp.139-140 (1960)

(12) J. A. Harley : "Advances in piezoresistive probes for atomic force microscopy", $\mathrm{PhD}$ dissertation, Standford Univ. (2002)

(13) A. J. Chapman : "Fundamentals of Heat Transfer" (1987)

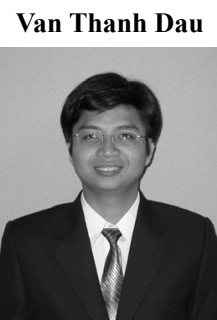

(Non-member) received the bachelor degree in Aeronautical Engineering from Hochiminh City University of Technology, Vietnam, in 2002. He received Master degree in Robotics from Ritsumeikan University, Japan, in 2004 and he is now pursuing the Doctor degree at Micro Nano Integrated Devices Laboratory, Ritsumeikan University. His current researches are $\mathrm{Si}$ based motion micro sensors and micro actuators.
Dzung Viet Dao (Member) received the bachelor degree in

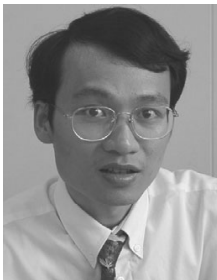
Informatics-Mechanical Engineering and master degree in Machinery Mechanics from Hanoi University of Technology (HUT) in 1995 and 1997, respectively. He has been a lecturer in Faculty of Mechanical Engineering of HUT since 1995. He was also serving as a mechanical engineer at Alcatel Telecom Company in Vietnam from 1995 to 1999.

In 1999 he entered the Ritsumeikan University, Japan, where he received the Ph.D degree in Science and Engineering in 2003. From 2003 to now he is a postdoctoral fellow at Micro Nano Integrated Devices Laboratory, Ritsumeikan University. His current interests are Si-based micro sensors and micro actuators.

Masahiro Hayashida (Non-member) was born Kumamoto, Japan in 1983.

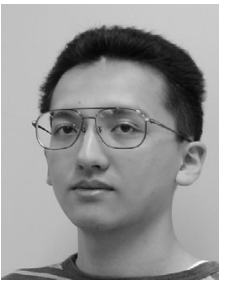
He received the B.E. degree in Science and engineering from Ritsumeikan University, Japan in 2005, respectively. $\mathrm{He}$ is currently working towards M.E. degree at Ritsumeikan University. His reasearch topics are thermal silicon accelerometer in MEMS integrated sensor systems.

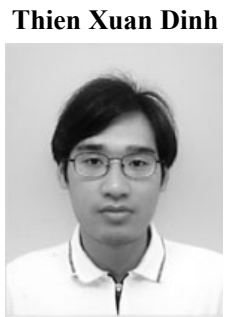

(Non-member) was born in 1979, in Vietnam.Undergraduate studied from 1997-2002 at HoChiMinh City University of Technology, Vietnam, graduating with a Bachelor of Engineering in Aeronautical Engineering. From 2002 to now attended Ritsumeikan University, graduating Master of Science degree in Fluid Mechanics in 2004, and now, joins the PhD in Fluid Mechanics course.

Susumu Sugiyama

(Member) received the B.S. degree in Electrical Engineering from Meijo University, Nagoya, in 1970, and the Dr. Eng. degree from Tokyo Institute of Technology, Japan, in 1994. From 1965 to 1995, he was with Toyota Central R\&D Laboratories, Inc., where he worked on silicon sensors, integrated sensors and micromachining. Since 1995 he has been with Ritsumeikan University, Shiga, Japan, where he has been serving as a professor in the College of Science and Engineering. He is now a professor in the Department of Micro System Technology and the director of the Research Institute for Micro System Technology at Ritsumeikan University. His current interests are MEMS, integrated sensor systems and synchrotron radiation 3-D lithography. 